\title{
CARACTERÍSTICAS CLÍNICO EPIDEMIOLÓGICAS DE LA INTOXICACIÓN POR PLAGUICIDAS Y ASOCIACIÓN ENTRE EDAD Y SEXO CON LA INTOXICACIÓN VOLUNTARIA HOSPITAL DE VITARTE 2013-2016
}

\author{
CLÍNICAL EPIDEMIOLOGICAL CHARACTERISTIC OF PESTICIDE POISONING AND ASSOCIATION BETWEEN AGE \\ AND SEX WITH VOLUNTARY INTOXICATION HOSPITAL OF VITARTE 2013 - 2016
}

Gustavo Alonzo Zapata-Coritoma', Jhony A. De La Cruz-Vargas ${ }^{1,2}$, Manuel Huamán-Guerreo

\begin{abstract}
RESUMEN
Objetivos: Determinar las características clínico epidemiológicas de la intoxicación por plaguicidas y la asociación entre edad y sexo con la intoxicación voluntaria en pacientes atendidos en el Hospital de Vitarte durante el periodo 2013- 2016. Métodos: Estudio observacional, retrospectivo, analítico. Incluidos 142 pacientes atendidos en el Hospital de Vitarte por intoxicación por plaguicidas, registrándose a partir de las historias clínicas, datos clínico epidemiológicos, asociándose dos de ellos (edad y sexo) a la intoxicación voluntaria. Resultados: La prevalencia y mortalidad fueron de $1,16 \%$ y $0 \%$, respectivamente. La población en un $63,4 \%(n=90)$ fue del sexo femenino, $42,3 \%$ $(n=60)$ adolescente, $64,1 \%(n=91)$ con nivel de instrucción secundaria, y 65,4\% ( $n=93)$ con ocupación laboral. El tipo de intoxicación en el $81,7 \%(n=116)$ fue voluntario, los insecticidas obtuvieron un $81,7 \%(n=116)$ y según su función $64,8 \%(n=92)$ estuvo expuesta a carbamatos. El 71,1\% presentó náuseas y vómitos, $52,8 \%(n=75)$ miosis y 46,5\% ( $n=66)$ dolor abdominal. Se halló asociación entre el sexo femenino y la intoxicación voluntaria ( $\times 2=6,1$; $\mathrm{p}=0,014 ; \mathrm{OR}=2,9 \mathrm{IC}: 1,2-6,9)$ y entre la edad adolescente y la intoxicación voluntaria $(x 2=19,9 ; \mathrm{p}=0,000 ; \mathrm{OR}=26,8$ IC:3,51-204,292). Conclusiones: Población caracterizada por ser predominantemente femenina y adolescente, prevaleciendo las intoxicaciones, de tipo voluntaria con insecticidas carbamatos provocando cuadro clínico caracterizado por afección del sistema nervioso central, digestivo, cardiaco, respiratorio y glándulas exocrinas. Existe asociación significativa entre el género femenino y la edad adolescente con la intoxicación voluntaria. Se recomienda evaluar el perfil psicológico y psiquiátrico de la población.
\end{abstract}

Palabras clave: Plaguicida; Intoxicación; Manifestaciones clínicas; Edad; Sexo; Ocupación; Instrucción. (fuente: DeCS BIREME)

\begin{abstract}
Objective: To determine the clinical epidemiological characteristics of pesticide poisoning and the association between age and sex with voluntary intoxication in patients treated at Vitarte Hospital during the period 20132016. Methods: Observational, retrospective, analytical study. Including 142 patients treated at the Hospital de Vitarte for pesticide poisoning. Clinical epidemiological data were recorded based on clinical records, two of them (age and sex) being associated with voluntary intoxication. Results: Prevalence and mortality were $1.16 \%$ and $0 \%$, respectively. The population in $63.4 \%(n=90)$ was female, $42.3 \%(n=60)$ adolescent, $64.1 \%$ $(n=91)$ with secondary education, and $65.4 \%(N=93)$ with employment. The type of intoxication in $81.7 \%$ $(n=116)$ was voluntary, the insecticides obtained $81.7 \%(n=116)$ and according to their function $64.8 \%(n$ = 92) was exposed to carbamates. $71.1 \%$ had nausea and vomiting, $52.8 \%(n=75)$ miosis and $46.5 \%(n=66)$ abdominal pain. There was an association between female sex and voluntary intoxication $(x 2=6.1, p=0.014$, $\mathrm{OR}=2.9 \mathrm{Cl}: 1.2-6.9)$ and between adolescent and voluntary intoxication $(x 2=19,9 ; \mathrm{p}=0.000 ; \mathrm{OR}=26.8 \mathrm{IC}: 3.51$ 204.292). Conclusions: Population characterized by being predominantly female and adolescent, prevailing intoxications, of a voluntary type with carbamate insecticides provoking a clinical picture characterized by affection of the central nervous system, digestive, cardiac, respiratory and exocrine glands. There is a significant association between the female gender and the adolescent age with voluntary intoxication. It is recommended to evaluate the psychological and psychiatric profile of the population.
\end{abstract}

Key words: Pesticide; Intoxication; Clinical manifestations; Age; Sex; Occupation; Instruction. (source: MeSH NLM)

\footnotetext{
INICIB, Facultad de Medicina Humana, Universidad Ricardo Palma.

${ }^{2}$ Instituto de Investigación en Ciencias Biomédicas, Universidad Ricardo Palma, Lima, Perú.
}

Correspondencia: Gustavo Alonzo Zapata-Coritoma. Dirección: Mz. D Lote 35 Urb. Los Angeles, Ate. Celular: 992619963 Correo: gustavozapata_533@outlook.com

Citar como: Gustavo Alonzo Zapata-Coritoma, Jhony A. De La Cruz-Vargas, Manuel Huamán-Guerreo. Características clínico epidemiológicas de la intoxicación por la plaguicidas y asociación entre edad y sexo con la intoxicación voluntaria, Hospital de Vitarte 2013 - 2016 [Artículo de Original]. Rev. Fac. Med. Hum. 2016;16(3):69-76. DOI 10.25176/RFMH.v16.n3.655 


\section{INTRODUCCIÓN}

La Organización de las Naciones Unidas para la Alimentación y la Agricultura (FAO) define a un plaguicida como una sustancia o mezcla de ellas dirigidas a prevenir, destruir o controlar cualquier plaga, incluyendo vectores que provoquen enfermedad en los humanos o animales ${ }^{1}$.

La OMS calcula que se registran entre 1 a 5 millones de casos de intoxicación por plaguicidas anualmente, América Latina aporta el $75 \%$ de los casos².

En el Perú 19 del total de 24 departamentos, han reportado casos de intoxicación aguda por plaguicidas, Lima con un 59,2\% (909 casos), Arequipa con un 10,4\% (160 casos), Junín con un $9,4 \%$ (144) y Piura con un $4,4 \%$ (68 casos) son los departamentos donde más frecuentemente se presenta este tipo de casos. Se ha notado que existen cuatro veces más riesgo de intoxicación por plaguicidas en el departamento de Piura y dos veces más en Arequipa, Junín y Lima³.

Es debido a su impacto y frecuencia, que en el $2014 \mathrm{EI}$ Ministerio de Salud del Perú, en el marco de las políticas sanitarias y de gestión pública, en el ejercicio de su rol rector, a través de la Dirección General de Epidemiología estableció la Norma Técnica de Salud NTS-109-MINSA/DGE-V.01 que establece la "Vigilancia Epidemiológica en Salud Pública del Riesgo de exposición e intoxicación por Plaguicidas aprobada mediante la RM No 649-2014/MINSA, con el fin de contribuir en la prevención y control de la exposición e intoxicación por plaguicidas en la población del Perú ${ }^{4}$

En el Hospital de Vitarte las intoxicaciones por plaguicidas según el ASIS del 2013 se encontraba como principal causa identificada de hospitalización, y en un segundo lugar entre las enfermedades sujetas a vigilancia Epidemiológica de notificación Individual según el ASIS del 2015, suponiendo un problema de salud Pública muy importante a abordar en la actualidad ${ }^{5,6}$.

\section{MÉTODOS}

Se realizó un estudio daservacional, retrospectivo y analítico. Observacional debido a que no hay intervención sobre la población, retrospectivo porque se hace una revisión en retrospectiva durante los años 2013 - 2016. Analítico porque se buscó asociación entre tres variables sometidas a estudio.

Metodología cuantitativay con la aplicación de una ficha de recolección de datos . La población está constituida por 142 pacientes con diagnóstico de intoxicación por plaguicidas (CIE-10: T60.0/T60.2/T60.3/T60.8/T60.9) en el servicio de Medicina y Pediatría del Hospital Vitarte durante el periodo 2013-2016 que cumplieron los criterios de inclusión y exclusión. Este estudio no fue necesaria la toma de muestra debido a que no hubo obstáculos en el tiempo ni procesamiento de datos que retrasen la realización de la misma. La unidad de análisis fueron todos los pacientes atendidos.

Los datos fueron recogidos a través de una ficha de recolección (tomada a partir de la Norma Técnica de la estrategia de vigilancia epidemiológica en Salud Pública del riesgo de exposición e intoxicación por plaguicidas del MINSA del año 2014), cuyos datos se basaron en los objetivos planteados en este trabajo de investigación, posteriormente se trasladó dichos datos a un papel en físico, luego se transcribió a hojas de cálculo del programa Microsoft Excel 2013. Posteriormente los datos se analizaron mediante el paquete estadístico SPSS.

Se realizó un análisis de la normalidad de las variables cuantitativas (año y edad) mediante el test de Kolmogorov-Smirnov el cual resultó con una $p<0,05$ por lo que se usaron pruebas no paramétricas y análisis descriptivo; chi cuadrado, frecuencia y porcentaje para las variables cualitativas, mientras que para las variables cuantitativas mediana y rango. Se analizó la fuerza de asociación entre el sexo femenino y edad adolescente con la intoxicación de tipo voluntaria.

El cálculo de la mortalidad se realizó en base a la fórmula de mortalidad específica para una enfermedad.

El cálculo de la prevalencia se realizó usando el programa Winepi tomando como nivel de confianza expresado como porcentaje, tamaño de población conocido, el origen de los datos de la población, la sensibilidad y especificidad perfectas y la prevalencia expresada como porcentaje.

\section{A. Programas a utilizar para análisis de datos}

Los programas a utilizar para el análisis de datos de este trabajo serán:

\section{- Microsoft Excel \\ - SPSS}

\section{Criterios de Inclusión:}

Todos los expedientes de pacientes con el diagnóstico de admisión de intoxicación por plaguicidas (CIE-10: T60.0/T60.2/T60.3/T60.8/T60.9) durante el periodo 2013- 2016. 


\section{Criterios de Exclusión:}

Historias Clínicas con datos incompletos.

Pacientes con diagnóstico de otro tipo de intoxicación además de plaguicidas

\section{RESULTADOS}

Seestudiaron 142 pacientes intoxicados por plaguicidas atendidos en el Hospital de Vitarte durante el periodo 2013 - 2016. Presentándose 0\% de mortalidad.

La tendencia de casos fue, en líneas generales, decreciente, 46,5\% $(n=66)$ en el año $2013,21,1 \%(n=30)$ el 2014, 15,5\% (n=22), y 16,9\% (n=24) el año 2016.

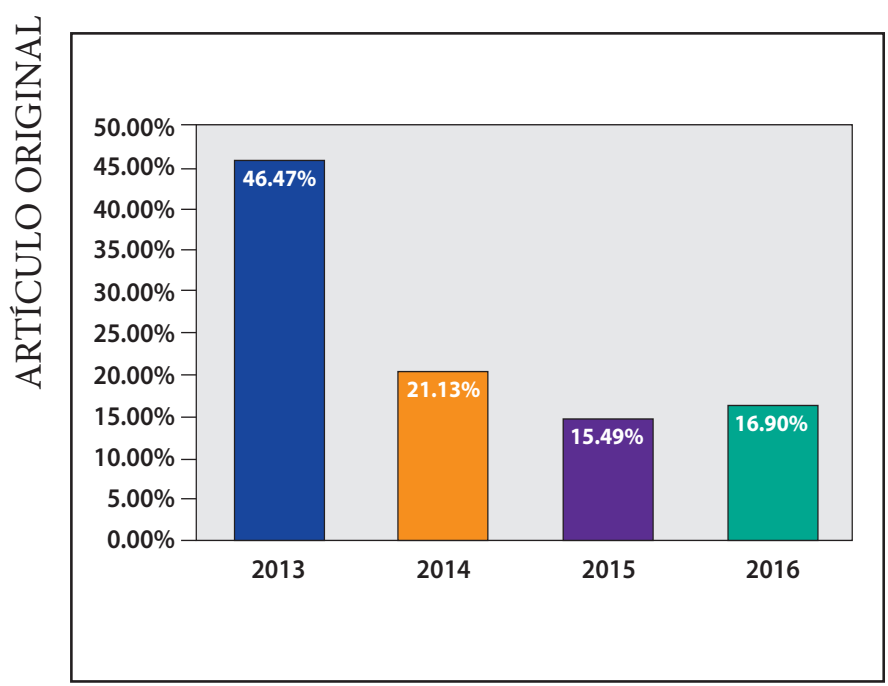

Gráfico 1. Porcentaje de pacientes según año atendidos en el Hospital de Vitarte durante el periodo 2013 - 2016. Fuente: INICIB-FAMURP/ Hospital Vitarte

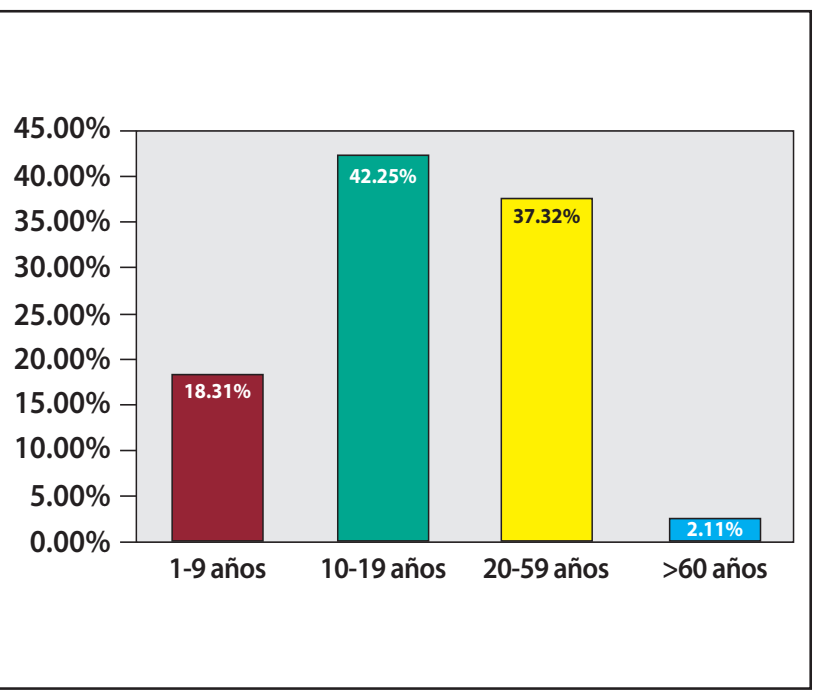

Gráfico 2. Porcentaje de pacientes según grupo etario atendidos por intoxicación por plaguicidas en el Hospital de Vitarte durante el periodo 2013 - 2016.

Fuente: INICIB-FAMURP/ Hospital Vitarte

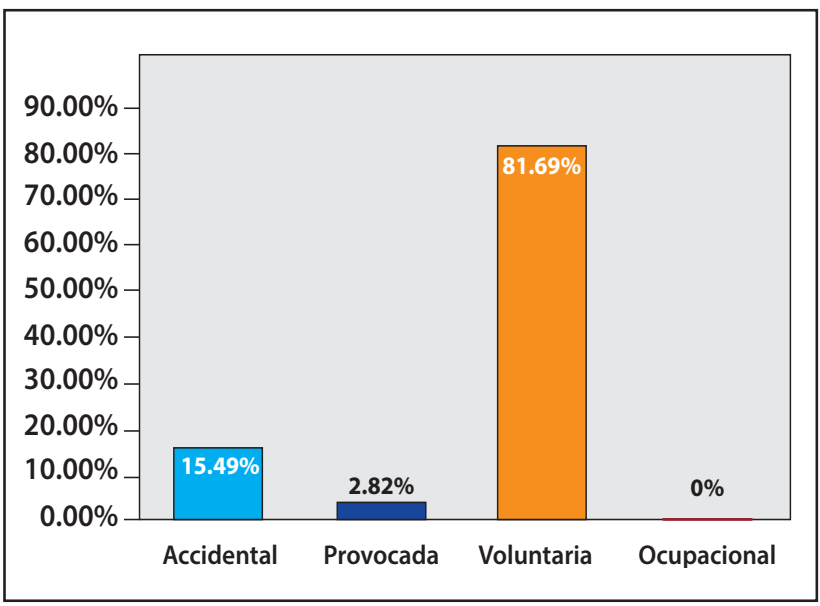

Gráfico 3. Porcentaje de pacientes según tipo de intoxicación atendidos por intoxicación por plaguicidas en el Hospital de Vitarte durante el periodo 2013 - 2016.

Fuente: INICIB-FAMURP/ Hospital Vitarte

Tabla 1. Frecuencia de pacientes según tipo de plaguicida atendidos por intoxicación por plaguicidas en el Hospital de Vitarte durante el periodo 2013 - 2016.

\begin{tabular}{lccc}
\hline \multicolumn{1}{c}{ Tipo de plaguicida } & \multicolumn{2}{c}{ Frecuencia } \\
\hline & Carbamatos & 92 & \\
\hline Insecticidas & Organofosforados & 5 & 126 \\
& Piretroides & 19 & \\
\hline Fungicida & - & - & 0 \\
\hline Herbicida & - & - & 0 \\
\hline Raticida & - & - & 26 \\
\hline Total & - & - & 142 \\
\hline
\end{tabular}

Fuente: INICIB-FAMURP/ Hospital Vitarte

Con respecto a las características epidemiológicas; el sexo femenino obtuvo una frecuencia de $63,4 \%(n=90)$ y el sexo masculino $46,6 \%(n=52)$. La frecuencia obtenida por la población adolescente fue de 42,3\% ( $n=60)$, seguido de la población adulta con $37,3 \%(n=53)$ de frecuencia, mientras que la población infante y adulta mayor tuvieron frecuencias de $18,3 \%(n=26)$ y $2,1 \%(n=3)$ respectivamente. La población con nivel de educación secundario fue de $64,1 \%(n=91)$, sin instrucción fueron $9,9 \%(n=14)$, con nivel primario 9,2\% $(n=13), 8,5 \%(n=12)$ con nivel inicial, y el mismo porcentaje lo obtuvieron las personas con nivel de instrucción superior. Las personas con alguna ocupación fueron $65,5 \% \quad(n=93)$ de la población y $36,6 \%(n=49)$ negó tenerla.

Con respecto a las características clínicas; de acuerdo al tipo de intoxicación, $81,7 \%(n=116)$ fueron voluntarias, $15,5 \%(n=22)$ accidentales, y 2,9\% $(n=4)$ provocada, no se registraron casos de intoxicación ocupacional. 
El tipo de plaguicida involucrado en un $64,8 \%(n=92)$ fueron los carbamatos, $13,4 \%(n=19)$ piretroides y $3,5 \%$ $(n=5)$ organofosforados, es decir, los insecticidas fueron responsables del $81,7 \%(n=126)$ de las intoxicaciones, mientras que los raticidas fueron responsables del $18,3 \% \quad(n=26)$ restante; no se observaron casos de intoxicación por herbicidas ni fungicidas. Finalmente las manifestaciones clínicas observadas fueron: náuseas y vómitos en un $75 \%(n=101)$ miosis en $52,8 \%(n=75)$, dolor abdominal en $46,5 \%(n=66)$, taquipnea en $39,4 \%$ $(n=56)$, sialorrea en $34,5 \%(n=49)$, fasciculaciones en $30,3 \%(n=43)$, trastorno de conciencia en $29,6 \%(n=42)$, taquicardia en $19,7 \%(n=28)$, debilidad muscular en $9,2 \%$ ( $n=13)$; incontinencia de esfínteres, disnea, diaforesis en $7 \%(n=10), 6,3 \%(n=9)$ y $4,6 \%(n=8)$ respectivamente; visión borrosa, bradicardia y mareos se observaron, cada uno de ellos en $4,9 \%(n=7)$ de los pacientes. Finalmente $1(0,7 \%)$ paciente presentó paro cardio-respiratorio y 1 $(0,7 \%)$ paciente no presentó síntomas.

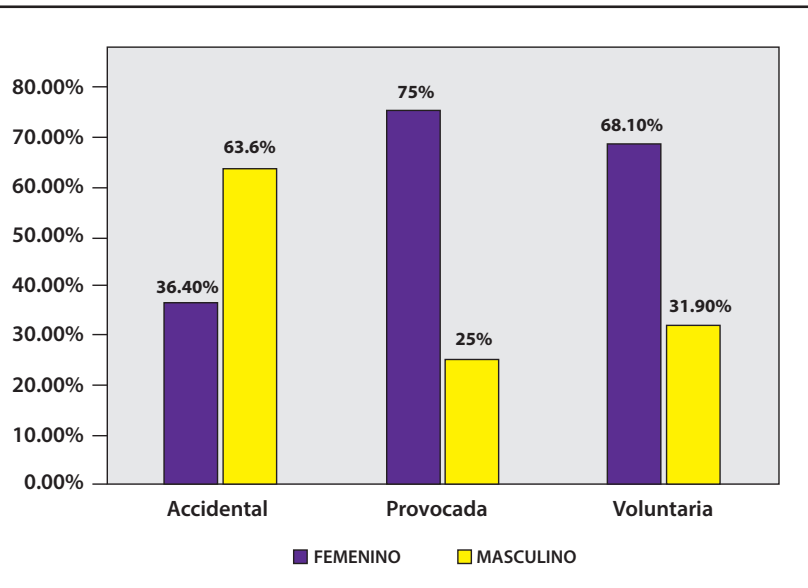

Gráfico 4. Porcentaje de pacientes según tipo de intoxicación y sexo atendidos por intoxicación por plaguicidas en el Hospital de Vitarte durante el periodo 2013 - 2016.

Fuente: INICIB-FAMURP/ Hospital Vitarte

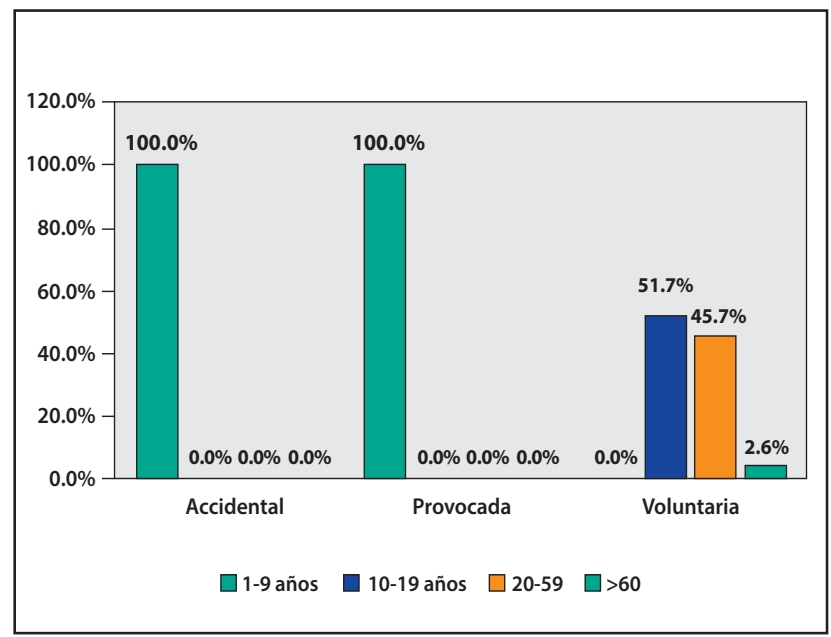

Gráfico 5. PPorcentaje de pacientes según tipo de intoxicación y grupo etario atendidos en el Hospital de Vitarte durante el periodo 2013 - 2016.

Fuente: INICIB-FAMURP/ Hospital Vitarte
El sexo femenino fue más frecuente en el tipo de intoxicación voluntaria y provocada con porcentajes de $68,10 \%(n=79)$ y $75 \%(n=3)$, respectivamente; mientras que el sexo masculino predominó en la intoxicación accidental con un $63,6 \%(n=14)$.

El sexo femenino predominó en los pacientes con intoxicación voluntaria con un porcentaje de $68,1 \%$ $(n=79)$, mientras que el sexo masculino, en los pacientes sin intoxicación voluntaria con un porcentaje de $57,7 \%$ $(n=15)$. La prueba de Chi cuadrado de Pearson determinó que el sexo está asociado significativamente $(p=0,014)$ con la intoxicación por plaguicidas en el género femenino. Así mismo, se determinó asociación entre el sexo femenino y la intoxicación voluntaria con una fuerza de asociación $\mathrm{OR}=6,089$.

Tabla 2. Tabla resumen de asociación de edad y sexo con la intoxicación voluntaria.

\begin{tabular}{lrrrr}
\hline \multicolumn{1}{l}{ Variables } & OR & IC $=95 \%$ & Significancia \\
$\begin{array}{l}\text { Sexo } \\
\text { Femenino }\end{array}$ & 6,089 & $1,219-6,953$ & 0,014 & $\mathrm{p}<0,05$ \\
$\begin{array}{l}\text { Edad } \\
\text { Adolescente }\end{array}$ & 26,786 & $3,512-204,292$ & 0,000 & $\mathrm{P}<0,05$ \\
\hline
\end{tabular}

Fuente: INICIB-FAMURP/ Hospital Vitarte

El $100 \%$ de pacientes con intoxicación de tipo accidental y provocada tenía edades entre 1 a 9 años, mientras que en la intoxicación voluntaria, el grupo etario comprendido entre 10 - 19 años tuvo un 51,7\% $(n=60)$, seguido de la población entre 20 a 59 años con $45,7 \%(n=53)$.

En la intoxicación voluntaria, prevaleció la población adolescente con un porcentaje de $51,7 \%(n=60)$.

La prueba de Chi2 determinó que la edad presenta asociación significativa $(p=0,000)$ con la intoxicación por plaguicidas en la edad adolescente. Así mismo se determinó asociación entre la edad adolescente y la intoxicación de tipo voluntaria con una fuerza de asociación OR = 19,869.

\section{DISCUSIÓN}

A nivel de las unidades que notifican a la DISA IV de Lima Este, los hospitales de dicha jurisdicción hasta el año 2015 son los que registran la mayor cantidad de casos de intoxicación por plaguicidas, ubicando al Hospital de Vitarte en el primer lugar?.

Nuestro estudio encontró tendencia decreciente de los casos durante el periodo estudiado, lo cual coincide con la tendencia que se ha observado en los últimos 7 años según la DISA a la cual corresponde?. 
Dos de las características epidemiológicas que más resaltan de la presente investigación, fueron : la mayor frecuencia del sexo femenino $(63,38 \%, n=52)$ y de la población adolescente $(42,25 \% \mathrm{n}=60)$; hallazgos que coinciden, en cuanto al género, con lo encontrado por Ener CJ y colaboradores ${ }^{8}$, Jiang $\mathrm{G}$ y colaboradores ${ }^{9} \mathrm{o}$ el realizado en nuestro país por Crisín $\mathrm{V}^{10}$. En lo que respecta al grupo etario, boletines epidemiológicos emitidos por el Centro Nacional de Epidemiología, Prevención y Control de Enfermedades (DGE) ${ }^{3}$ coinciden con nuestros resultados, al igual que los estudios realizados por Hernández Gonzales MM y colaboradores ${ }^{11}$ y Cepeda Ureña AF et.al ${ }^{12}$. Sin embargo, la literatura es clara en demostrar lo contrario, estudios como el de Pérez Rogriguez S. ${ }^{13}$, Varona $M$ et al. ${ }^{14}$ Hernandez Gonzáles MM y colaboradores ${ }^{11}$, Zúñiga VCM y Calderon $M^{15}$ Cepeda Urueña AF et al. ${ }^{12}$, e inclusive la DGE en nuestro país ${ }^{3}$ señalan que el género masculino es el más frecuentemente implicado en la intoxicación por plaguicidas, y otros como el de Barco JC y colaboradores ${ }^{16}$, Tuñiga VCM y Calderon MM $^{15}$, Villena J y colaboradores ${ }^{17}$, nos señalan claramente que la población mayormente expuesta es la que se encuentra en edades por encima de lo hallado por esta investigación, inclusive a lo hallado por la DGE en nuestro País o lo señalado por la DISA IV de Lima Este, en su último Análisis Situacional de Salud, en donde la población con mayor frecuencia de intoxicación por plaguicidas es la femenina y la que se encuentra entre los 18 y 29 años $^{7}$. Estas diferencias pueden ser explicadas al tomar en cuenta otras características epidemiológicas; por ejemplo, la actividad agrícola, que en nuestro país representa la actividad principalmente involucrada en la intoxicación por plaguicidas según el $\mathrm{DGE}^{3}$, ésta característica predominó, también en algunos estudios ${ }^{13,11,15,12}$; lo que podría explicar la mayor frecuencia del sexo masculino en estas poblaciones; a excepción de un estudio ${ }^{12}$ en el cual a pesar de que el sexo masculino fue la más frecuente, los sujetos no se dedicaban a dicha actividad. En lo que respecta a la edad es importante considerar el contexto geográfico, por ejemplo estudios realizados en Europa ${ }^{16}$ obviamente tendrán estadísticas de grupos etarios más longevos que la nuestra, o el contexto social de la población estudiada, es decir, si ésta es económicamente activa, tendrán edades superiores a los 18 años en su mayoría, pero queda en la palestra la razón por la cual los demás estudios a pesar de tener características casi idénticas con nuestra población, no tienen predominancia del género femenino ni de adolescentes, aún más cuando existen estudios en donde se abordó la intoxicación adolescente y no se encontró predominancia de éste género ${ }^{18}$.
En nuestra población la intoxicación voluntaria fue la de mayor frecuencia $(81,69 \% ; n=116)$, seguida de la accidental (15,49\%; $n=22)$, hallazgos que coinciden con estudios como el de Barco JC et al. ${ }^{16}$, Villar FJC et al. ${ }^{19}$, Exner $\mathrm{CJ}^{8}$, Cepeda Urueña et al. ${ }^{12}$.Ésta variable respondería a la pregunta planteada en el párrafo anterior, ya que en nuestro estudio el sexo femenino y la edad adolescente se encontraron con mayor frecuencia en pacientes con intoxicación voluntaria lo cual se ve apoyado por estudios como el de Chen $Z$, et al. ${ }^{20}$ y el de Zhao et al. ${ }^{21}$,y Masot Rangel A., en Cuba $^{22}$. Así mismo, en nuestro estudio se pudo demostrar asociación significativa $(p=0,0014)$ entre el sexo femenino y la intoxicación de tipo voluntaria, además de que se determinó como un factor de riesgo asociado a la intoxicación voluntaria $(\mathrm{OR}=2,912$ $I C=1,219-6,953)$, probablemente porque el sexo femenino se encuentra más propenso a presentar tendencias tanáticas o autolesivas.

En cuanto al análisis de asociación entre edad e intoxicación voluntaria la presente investigación encontró que la edad adolescente es un factor de riesgo asociado a intoxicación voluntaria con un OR de 19,869 estadísticamente significativo $p=0,000$ pero con un amplio intervalo de confianza (IC: 3,5-204,3) el cual, tiene que ser abordado en estudios posteriores. Lo anteriormente dicho coincide con estudios realizados como el de Rubinos RR y colaboradores 23, en donde se halló un $\mathrm{p}=0,0000$ al $95 \%$ de confianza para las variables edad adolescente y tipo de intoxicación.

Un punto importante que no debe pasar desapercibido es que en nuestro estudio no se reportaron casos de intoxicación ocupacional, como sí sucedió en otros estudios ${ }^{11,15}$ donde inclusive éste tipo de intoxicación fue el más frecuente siguiendo la tendencia de nuestra realidad según los boletines emitidos por la $\mathrm{DGE}^{3}$.

La mortalidad y prevalencia obtenida en nuestro estudio fueron de $0 \%$ y 1,16\%, respectivamente; ambos hallazgos distan de estudios como el de Aveiga Valdivieso AA y colaboradores ${ }^{24}$ cuya prevalencia hallada fue de $0,05 \%$ o el de Cepeda Urueña AF y colaboradores 12 en la que se halló una prevalencia de 0,014\%, y cuya mortalidad fue del 5\%, \% o el de Gómez Villegas $C D^{25}$, en la que la prevalencia encontrada fue de $0,11 \%$ mientras que la mortalidad correspondió al $1,59 \%$ inclusive durante un periodo de tiempo igual al abordado en nuestro estudio. Esta discordancia puede ser explicada, en el caso de la prevalencia, a las características epidemiológicas antes descritas (edad, sexo, tipo de población, y sobre todo el tipo de intoxicación); en cuanto a la notable diferencia en 
la mortalidad, ésta puede explicarse al tiempo que le toma a los pacientes acudir a los servicios de salud, y la cantidad, tipo y hasta composición química del plaguicida involucrado, variables que deberían ser abordadas en estudios posteriores.

En lo que respecta al grado de instrucción el nivel secundario fue el que predominó $(64,08 \% ; n=91)$; siendo las personas sin instrucción, las que ocuparon el segundo grupo en frecuencia $(9,86 \% ; n=14)$, datos que concuerdan parcialmente con lo hallado por Cepeda Urueña AF y colaboradores ${ }^{12}$, ya que el $36,8 \%$ $(n=100)$ de pacientes en este estudio tenía como grado de instrucción el nivel secundario, sin embargo, la población sin instrucción fue solo del 8,5\% ( $n=23)$, representando la minoría en este estudio.

En nuestra población la frecuencia de pacientes con ocupación fue del $65,49 \%$, lo cual también dista de estudios como el de Ramos Hernández ${ }^{26}$ en donde el mayor porcentaje de pacientes no tenía ocupación alguna, este nivel de contraste resulta particular en nuestra población, ya que aparentemente el tener el tiempo ocupado en alguna actividad no repercute en la intoxicación por plaguicidas, como así lo sugiere Coronado Molina ${ }^{27}$, un estudio realizado en nuestra realidad.

Con respecto al tipo de plaguicida nuestro estudio encontró que los insecticidas fueron los de mayor frecuencia $(81,69 \% ; n=126)$, lo cual coincide con los boletines epidemiológicos emitidos hasta el $2015^{3}$ y con estudios como el de Jian $\mathrm{G}$ y colaboradores ${ }^{9}$ donde los insecticidas obtuvieron un porcentaje de $86,7 \%$. El segundo plaguicida de mayor frecuencia fueron los raticidas (18,31\%; $n=26)$, lo que no coincide con el estudio anteriormente mencionado, ya que en dicho estudio éste tipo de plaguicida obtuvo un tercer lugar en frecuencia con un porcentaje de 3,6\% por debajo del 5,1\% obtenido por los herbicidas, plaguicida no observado en nuestro estudio. En nuestro trabajo de investigación los insecticidas carbamatos fueron los que se observaron con mayor frecuencia (64,80\%; $n=92)$, coincidiendo con el estudio de Cepeda Urueña AF et al. ${ }^{12}$, en donde los tóxicos principalmente usados para las intoxicaciones fueron los carbamatos con un 62,1\%, sin embargo, la gran mayoría de estudios revisados para este trabajo de investigación refieren a los insecticidas organofosforados como los que se ubican en primer lugar de frecuencia con porcentajes, por ejemplo , de $50 \%{ }^{16}, 44 \%{ }^{11}, 58 \%{ }^{17}, 70,6 \%^{20}$ y otros ${ }^{28}$. Esta discordancia con respecto a este trabajo probablemente se deba a la composición química usada para la fabricación de los plaguicidas, ya que en la práctica diaria se observó que los pacientes se refieren a los agentes que producen sintomatología colinérgica, como "raticidas", debido a que, el producto que en su mayoría se ha visto involucrado ("Campeón") es comercializado como tal.

Con respectoala sintomatologíalosmásfrecuentemente observados fueron: náuseas y vómitos, miosis, dolor abdominal, taquipnea, sialorrea, y fasciculaciones, lo que coincide con los hallazgos de Exner CJ y colaboradores ${ }^{8}$, o el realizado por Crispín y colaboradores ${ }^{10}$. Podríamos resumir de forma práctica que, los síntomas digestivos fueron los más frecuentemente observados, así como el estudio de Villar FJC y colaboradores ${ }^{19}$.

Este trabajo surgió de la observación empírica del comportamiento de las características clínica epidemiológicas en la población ya mencionada, da a conocer una realidad alarmante, sobre todo en la población femenina adolescente, la cual como ha quedado demostrado líneas atrás, contrasta significativamente con otras poblaciones. Por ello su importancia y relevancia, porque es de los pocos estudios encontrados en la bibliografía nacional y mundial que, además de demostrar asociación entre las variables sexo y edad con la intoxicación voluntaria, mide la fuerza de asociación entre ellas.

Finalmente, este trabajo no solo aporta nuevos conocimientos, sino también generan preguntas de investigación que deberán ser evaluadas en futuras estudios, por ejemplo: ¿por qué en una población en la que predomina el tipo de ocupación industrial no se presentaron intoxicaciones ocupacionales? ¿Por qué la nula mortalidad, inclusive en 4 años de estudio? ¿Cuál es la relación entre el resto de variables con la intoxicación voluntaria en la población femenina adolescente? ¿Por qué la contundente predominancia del género masculino en la intoxicación accidental?.

\section{CONCLUSIÓN}

Las características clínicas más frecuentemente encontradas fueron el tipo de exposición voluntaria (81,69\%; $n=116)$, los insecticidas (81,69\%; $n=126)$ y carbamatos $(64,80 \% ; n=92)$; según el tipo y función de plaguicida respectivamente. Finalmente, las manifestaciones clínicas que prevalecieron fueron: Náuseas y, Vómitos $(71,1 \% ; n=101)$, miosis $(52,8 \%$; $\mathrm{n}=75$ ) y dolor abdominal $(46,5 \% ; n=66)$, de los pacientes intoxicados por plaguicidas del Hospital de Vitarte atendidos durante el periodo 2013 -2016

Las características epidemiológicas más frecuentemente encontradas fueron el sexo femenino $(63,4 \% ; n=52)$, la edad adolescente $(42,3 \% ; n=60)$, la presencia de 
ocupación (65,5\%; n=93)n y el grado de instrucción secundario $(64,1 \% ; n=91)$ en los pacientes intoxicados por plaguicidas del Hospital de Vitarte atendidos durante el periodo 2013 -2016.

El sexo femenino y la edad adolescente están asociados significativamente con $\mathrm{OR}=6,1 \mathrm{p}=0,0014$ y $\mathrm{OR}=26,786$, $\mathrm{p}=0,000$ respectivamente, a la intoxicación de tipo voluntaria por plaguicidas en pacientes atendidos en el Hospital de Vitarte durante el periodo 2013- 2016.

La prevalencia encontrada fue de 1,16\%, lo que supera con creces a otras realidades revisadas para este estudio. Por otro lado se halló una mortalidad de $0 \%$, lo cual resultó ser la más baja con respecto a todos los estudios revisados para este trabajo de investigación.

La edad más frecuente en la intoxicación de tipo accidental y provocada fueron los pacientes de edad pediátrica, mientras que la intoxicación voluntaria fue el único tipo de intoxicación en los adolescentes, adultos y adultos mayores intoxicados por plaguicidas del Hospital Vitarte atendidos durante el periodo 2013- 2016.

No se presentaron casos de intoxicación ocupacional, ni de intoxicación por fungicidas o herbicidas en pacientes intoxicados por plaguicidas del Hospital Vitarte atendidos durante el periodo 2013- 2016.

Financiamiento: Autofinanciado.

Conflicto de interés: Los autores declaran no tener conflictos de interés en la publicación de este artículo.

Recibido: 29 de diciembre del 2016

Aprobado: 26 de enero del 2017

\section{REFERENCIAS BIBLIOGRÁFICAS}

1. Dufol AF. Intoxicación por plaguicidas. En: Anales del Sistema Sanitario de Navarra [Internet]. 2003 [citado 22 de junio de 2016]. p. 155-171. Disponible en: http://recyt.fecyt.es/index.php/ASSN/article/ viewArticle/5017

2. Demográficas T. de Mortalidad en la Región de las Américas, 19802000. Bol Epidemiológico Organ Panam Salud. 2002;23(3).

3. Anibal Velásquez Valdivia. Boletín Epidemiológico [Internet]. Lima: Dirección General de Epidemiología; 2015 [citado 21 de junio de 2016]. Report No.: 39. Disponible en: http://www.dge.gob.pe/portal/docs/ vigilancia/boletines/2015/39.pdf

4. Norma Técnica de Salud que establece la Vigilancia Epidemiológica en Salud Pública del Riego de Exposición e Intoxicación por Plaguicidas. NTS No 109 - MINSA/DGE - V 012014 p. 26.

5. Magdalena Gladys Bazán Lossio. ASIS Hospital de Vitarte 2013 [Internet]. Lima, Perú: MINSA; 2013. Report No.: Final. Disponible en: http://www.hospitalvitarte.gob.pe/portal/mod/transparencia/index. php?transparencia $=778$

6. Cesar Agusto Conche Prado. ASIS Hospital de Vitarte 2015 [Internet] Lima, Perú: MINSA; 2015. Report No.: Final. Disponible en: http:// www.hospitalvitarte.gob.pe/portal/mod/transparencia/index. php?transparencia $=778$

7. Anibal Velásquez Valdivia. Análisis de Situación de Salud de la DIrección de Salud IV Lima Este - 2015 [Internet]. Lima, Perú: MINSA; 2015 p. 320. Disponible en: http://www.limaeste.gob.pe/limaeste/DIRECCIONES/OEP/ Asis/SemanasEpi/Analisis\%20de\%20Situacion\%20de\%20Salud/2015/ ASIS_DISA_IV_LE_\%202015\%20-\%20Preliminar.pdf

8. Exner CJ, Guillermo Urquizo A. Intoxicación por organofosforados: experiencia institucional. Cuad Hosp Clínicas. 2007;52(1):60-3.

9. Jiang G, Lian L, Wang C, Li M, Wang J, Pan N, et al. [The characteristics and trends of acute pesticide poisoning of Shaoxing in 2006 to 2011]. Zhonghua Lao Dong Wei Sheng Zhi Ye Bing Za Zhi Zhonghua Laodong Weisheng Zhiyebing Zazhi Chin J Ind Hyg Occup Dis. julio de 2013;31(7):511-3.
10. Crispín V, Crispín PH, Abarca F, Almonacid RD. Intoxicación aguda por carbamato en el Centro de Salud Miguel Grau Chaclacayo Lima 20032007. Ciencia e Investigación. 2008;11(2):29-34.

11. Hernández González MM, Jiménez Garcés $C$, Jiménez Albarrán FR, Arceo Guzmán ME. Caracterización de las intoxicaciones agudas por plaguicidas: perfil ocupacional y conductas de uso de agroquímicos en una zona agrícola del Estado de México, México. Rev Int Contam Ambient. 2007;23(4):159-167.

12. Cepeda Urueña AF, Jeréz Cantor WR, Ramírez Orjuela RD, Franco Zambrano D, Hurtado Velásquez VP, Ortiz Plazas GA. Caracterización sociodemográfica de casos de intoxicación por plaguicidas en el período comprendido entre el 1 enero de 2010 y el 31 de diciembre de 2011, registrados en los servicios de urgencias del hospital San Rafael de Facatativá [Internet] [Tesis Pregrado Médico Cirujano]. [Facultad de Ciencias de la Salud]: Universidad de Ciencias Aplicadas y Ambientales; 2012 [citado 7 de febrero de 2017]. Disponible en: http://trafficlight. bitdefender.com/info?url=http\%3A//repository.udca.edu.co\%3A8080/ jspui/handle/11158/124\&language=en_US

13. Pérez Rodríguez S, Álvarez Delgado M, Baldo MD, Capote Marrero B. Intoxicaciones agudas por plaguicidas consultadas al Centro Nacional de Toxicología durante el bienio 2007-2008. Rev Cuba Med Mil. 2012;41(4):415-422.

14. Varona M, Morales L, Ortíz J, Sánchez JF, Cárdenas O, De la Hoz F. Panorama epidemiológico de exposición a plaguicidas inhibidores de colinesterasa en 17 departamentos del país. Biomédica. 1998;18(1):22-9.

15. Zuñiga VCM, Calderon MM. Revisión de los casos de intoxicaciones con plaguicidas atendidos en el Hospital Max Peralta Cartago, Costa Rica (1978-1983). Rev Costarric Cienc Med. 1986;7(2):191-5.

16. Barco JC, Omar C, Puiguriguer J, Coll I, Barcelo B, Castanyer T. Revisión de las intoxicaciones graves por insecticidas organofosforados atendidas en un período de 11 años (1996-2006). Emergencias [Internet]. 2008 [citado 22 de junio de 2016];20(3). Disponible en: https://www. researchgate.net/profile/Jordi_Puiguriguer/publication/28221089_ Revision_de_las_intoxicaciones_graves_por_insecticidas organofosforados_atendidas_en_un_periodo_de_11_anos_1996-2006/ links/5469f1670cf2397f782f7124.pdf 
17. Villena J, Córdoba A, Peñaloza de Terán M. Intoxicaciones con Plaguicidas en San Miguel de Tucumán durante el período 2001-2002. Bol Electrónico Mens Serv Toxicol Sanat Niños Rosario Ar. 2005;2(36):1-15.

18. Rubinos RR, Rodríguez SP, Oñoz NG, de León Consuegra JP. Intoxicaciones agudas en la adolescencia. Rev Arch Méd Camagüey [Internet]. 2014 [citado 15 de febrero de 2017];12(2). Disponible en: http://trafficlight.bitdefender.com/info?url=http\%3A//revistaamc.sld.cu/ index.php/amc/article/view/2602\&language=en_US

19. VILLAR FJC, CAMACHO AXA, SOTO GAC. Características clínicas sociodemográficas de las intoxicaciones en los niños de 1 a 17 años de edad del Hospital Universitario Hernando Moncaleano Perdomo en un período comprendido entre noviembre de 2001 a octubre de 2002 / Francisco Javier Caldon Villar, Angela Ximena Argote Camacho, Guillermo Alberto Casanova Soto [Internet] [Tesis Pregrado Medico Cirujano. Colombia]. [Facultad de Ciencias de la Salud]: Universidad Surcolombiana; 2004 [citado 22 de junio de 2016]. Disponible en: https://contenidos.usco. edu.co/images/documentos/grados/T.G.Medicina/173.T.G-FranciscoJavier-Caldon-Villar,-Angela-Ximena-Argote-Camacho,-Guillermo$\exists$ Alberto-Casanova-2004.pdf

20. Chen Z, Li S, Wang Y, Yu L, Yi G, Yan Y. Analysis of 4713 cases of Wuhan pesticide poisoning reports of year 2002 to 2010. Chin J Ind Hyg Occup Dis. octubre de 2011;29(10):776-8.

21. Zhao S, Liu S. [Epidemiological analysis for Quzhou pesticide poisoning of year 2008 to 2010]. Zhonghua Lao Dong Wei Sheng Zhi Ye Bing Za Zhi Zhonghua Laodong Weisheng Zhiyebing Zazhi Chin J Ind Hyg Occup Dis. septiembre de 2012;30(9):676-8.

22. Masot Rangel A, Fonseca Hernández M, Rodríguez Herrera E, Corona Martínez LA. Morbilidad por intoxicaciones agudas en el Hospital Pediátrico de Cienfuegos durante el quinquenio 1996-2000. Rev Cuba Pediatría. 2004;76(3):0-0.
23. RubinosI RR, Rodríguezll SP, García N. Acute intoxications in the adolescence. 2008 [citado 18 de septiembre de 2015]; Disponible en: http://scielo.sld.cu/pdf/amc/v12n2/amc02208.pdf

24. Aveiga Valdivieso A. Aplicación de un protocolo de manejo de intoxicaciones agudas por plaguicidas. Area de emergencias del Hospital del Niño« Francisco Icaza Bustamante», 2010 [Internet] [Tesis Magister en Emergencias Médicas. Ecuador]. [Facultad de Ciencias Médicas.]: Universidad de Guayaquil.; 2012 [citado 8 de febrero de 2017]. Disponible en: http://trafficlight.bitdefender.com/info?url=http\%3A//repositorio. ug.edu.ec/handle/redug/1079\&language=en_US

25. Gómez Villegas CD. Prevalencia de intoxicaciones por órganofosforado asociado a intento autolítico en usuarios as atendidos en el Hospital Regional Isidro Ayora de Loja durante el periodo 20092013 [Internet] [Tesis Médico General. Ecuador]. [Área de La Salud Humana]: Universidad Nacional de Loja; 2015 [citado 8 de febrero de 2017]. Disponible en: http://trafficlight.bitdefender.com/info?url=https\%3A//dspace.unl.edu. ec/jspui/handle/123456789/13466\&language=en_US

26. Ramos Hernández U de J. Aplicación de las normas terapéuticas en pacientes intoxicados por plaguicidas, servicio de emergencias Hospital Doctor Roberto Calderón Gutierrez, Managua 2008-2009 [Internet] [Tesis Especialista. Nicaragua]. [Facultad de Medicina Humana]: Universidad Nacional Autónoma de Nicaragua; 2010 [citado 3 de febrero de 2017]. Disponible en: http://trafficlight.bitdefender. com/info?url=http\%3A//pesquisa.bvsalud.org/enfermeria/resource/ es/lil-592882\&language=en_US

\section{Indizado en: latindex}

http://www.latindex.org/latindex/ficha?folio $=14280$<smiles>C1CC2CC1C2</smiles>

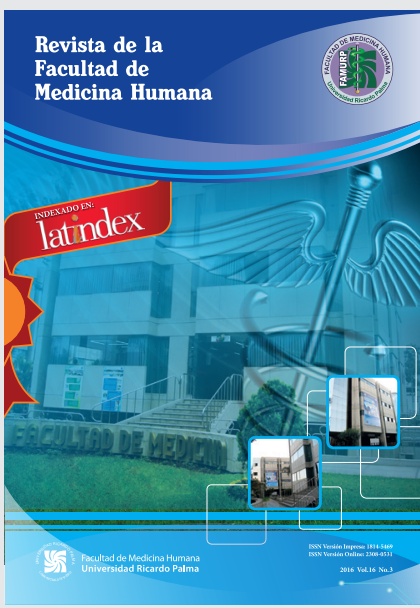

